\title{
Single-Bit Parity Detection and Correction using Hamming Code 7-Bit Model
}

\author{
Wirda Fitriani \\ Faculty of Computer Science \\ Universitas Pembangunan \\ Panca Budi \\ Jl. Jend. Gatot \\ Subroto Km. 4,5 \\ Sei Sikambing, \\ 20122, Medan, \\ Sumatera Utara, Indonesia
}

\author{
Andysah Putera \\ Faculty of Computer Science \\ Universitas Pembangunan \\ Panca Budi \\ Jl. Jend. Gatot Subroto \\ Km. 4,5 Sei Sikambing, \\ 20122, Medan, \\ Sumatera Utara, Indonesia
}

\author{
Utama Siahaan \\ Faculty of Computer Science \\ Universitas Pembangunan \\ Panca Budi \\ Jl. Jend. Gatot Subroto Km. \\ 4,5 Sei Sikambing, \\ 20122, Medan, Sumatera \\ Utara, \\ Indonesia
}

\begin{abstract}
In the world of technology is already integrated into the network must have a data transmission process. Sending and receiving data communications systems do not avoid mistakes. Packets of data sent from the server to the client computer always have an error in transmission. These shipments have leaks that occur due to changes in voltage, frequency or impact. One of the methods used to detect and correct errors in data transmission is the Hamming method. This method will check bit errors in delivery. Hamming is to do the process at fault detection, and then the error will be corrected so that the arrangement of the bits will go back to the bit sequence before the data is sent. With the application of this method, the data transmission process will avoid mistakes. Data will be saved to the destination.
\end{abstract}

\section{General Terms}

Data Transmission

\section{Keywords}

Hamming Code, Error Detection

\section{INTRODUCTION}

Delivery of a message from someone that is confidential or personal to others or the desired person is a way to change the message to be sent into a code word so the secret message can be read only by the sender and the person concerned. The theory is used to change a secret message into a code word or otherwise change the code word becomes the message is the theory Encoding [1]. Encoding is the process of adding binary numbers of $n-m$ characters in each code to form a code word. Decoding is the process of outlining a code word into a message [7][8]. The examples of encoding and decoding techniques are repetition and Hamming. In the encoding form of repetition code, the code word obtained by repeating a message that has been modified in the form $\mathrm{k}$ times as much code. In Hamming encoding, the code word is obtained by adding the binary numbers on every message that has been modified as $\mathrm{K}$ characters [9][10]. Meanwhile, in the form of repetition code decoding, the message is obtained by dividing the total code word $\mathrm{K}$ parts. In decoding such as Hamming code, the message was obtained by deleting the code word before by $\mathrm{K}$ character. To determine the received message is equal to sent it needs to be checked, and the error correcting code word is received, i.e., the specific ways to obtain the actual message.

\section{THEORIES}

\subsection{Data Transmission}

The data transmission is the process of sending data from one data source to a data receiver using an electronic device. To perform data transmission requires the medium, this medium itself has several kinds such as buses, cables commonly found on the computer, while the external computer in the transmission of data can use external cables (Wired) and Wi-Fi (Wireless).

\section{Wired Cable}

Wired cable is used to perform the data transmission process directly; there are several kinds of which are as follows:

- Wired UTP or commonly known as Unshielded Twisted Pair cable is used for transmitting over a computer network such as in the office or cafe. In addition to UTP, STP (Shielded Twisted Pair) in which there are several wires in one bundle can also be used to transmit data.

- Coaxial cable which consists of two kinds of conductors separated by using an insulator.

- Fiber Optics is commonly called FO, where a cable that can transmit information by passing data using light waves.

Wireless

Wireless communication is having telecommunications using electromagnetic waves instead of cable media. Nowadays wireless technology has developed rapidly. It can be seen by the increasing number of cellular phone use, besides developing wireless technology is also used for integrated into the same network. Wireless, also known as Media Transmission Wireless is unguided, can only transmit data without too much obstacles. The cast of data on the network is typically done using a tool known as the antenna or transceiver. Some of the wireless are:

- Radio

- Microwave

- Infra Red

- Bluetooth

- $\quad \mathrm{NFC}$ 


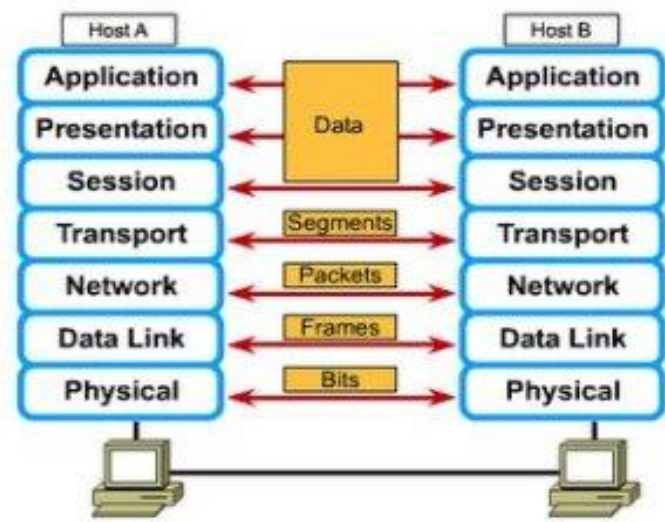

Fig. 1 Transmission Process

Figure 1 shows the OSI model. It is a description for layered communications and computer network protocol design. It was developed as part of the Open Systems Interconnection (OSI) initiative. In its most basic form, it divides network architecture into seven layers which, from bottom to top, are the Physical, Data-Link, Network, Transport, Session, Presentation, Application Layers.

\subsection{Hamming Code}

Hamming code is an error correction system that uses parity bits to correct the mistake [3][6]. Beside Hamming Code, there are many other systems in a more efficient error correction method to correct data error consists of many bits [4][5]. Parity of method is to check the the codes that exist. Linear error-correction code has various limitations error. On Hamming Code, only one mistake can be repaired. For a twobit mistake, it can be only detected.

Hamming method is a frequently used error detection and correction method. This method is quite simple. This method uses XOR logic operation in the detection and correction of errors. The tested data is a row of binary numbers of data transmitted. Hamming code will check the error from the first to the last bit, so the method is also called a linear error correcting code. Hamming code method works by inserting several pieces of check bits to data at a specific position. The number of check bits inserted depending on data length.

Where:

$$
c b=2^{n}-(n-1)>p
$$

cb : checkbit value

n : total checkbits

$\mathrm{p} \quad$ : bit length

Table 1. Check Bit and Total Bit

\begin{tabular}{|c|c|c|}
\hline Check-Bit & Total Bit & Position \\
\hline 1 & 0 & 1 \\
\hline 2 & 1 & 2 \\
\hline 3 & 4 & 4 \\
\hline 4 & 11 & 8 \\
\hline 5 & 26 & 16 \\
\hline 6 & 57 & 32 \\
\hline 7 & 120 & 64 \\
\hline 8 & 247 & 128 \\
\hline
\end{tabular}

Table 1 shows the limit of bit length. The total check-bits obtained depends on the bit length. For example, if the bit length is seven then the check-bit is 11. The following illustration will describe how it works.

$$
\begin{aligned}
\mathrm{p} & =7 \\
\mathrm{cb} & =2^{n}-(n-1)>7 \\
& =2^{1}-(1-1) \\
& =0<7 \\
\mathrm{cb} & =2^{n}-(n-1)>7 \\
& =2^{2}-(2-1) \\
& =1<7 \\
\mathrm{cb} & =2^{n}-(n-1)>7 \\
& =2^{3}-(3-1) \\
& =4<7 \\
\mathrm{cb} & =2^{n}-(n-1)>7 \\
& =2^{4}-(4-1) \\
& =11>7
\end{aligned}
$$

The calculation run continuously until reach $\mathrm{cb}>\mathrm{p}$. For bit length $=7$, there will be four check-bits to check the bit data.

Table 2. Bit example

\begin{tabular}{|l|l|l|l|l|l|l|}
\hline 1 & 0 & 0 & 1 & 0 & 0 & 0 \\
\hline 1 & 2 & 3 & 4 & 5 & 6 & 7 \\
\hline
\end{tabular}

Table 3. Data and parity

\begin{tabular}{|c|c|c|c|c|c|c|c|c|c|c|}
\hline $\mathbf{x}$ & $\mathbf{x}$ & 1 & $\mathbf{x}$ & 0 & 0 & 1 & $\mathbf{x}$ & 0 & 0 & 0 \\
\hline 1 & 2 & 3 & 4 & 5 & 6 & 7 & 8 & 9 & 10 & 11 \\
\hline
\end{tabular}

Table 2 shows the original data consists of seven bits only. When it is transmitted, the Hamming code adds some extra bits to mark the position. Since the bit length is seven bits, the result will be 11 bits as shown in Table 3 .

\section{METHODOLOGY}

Normally, a frame consists of $\mathrm{m}$ bits of data and $\mathrm{r}$ redundant, or check bits. Take the total length of $n(n=m+r)$. An n-bit unit containing data and check-bit often attributed as the $n$ bit code word. In most data transmission applications, all $2 \mathrm{~m}$ data messages are legal data. However, as regards the method of calculating the check bits, not all $2 \mathrm{n}$ used. If specified algorithm to calculate the check bits, it will be possible to make a complete list of the legal codewords. From this list can be searched two codewords its minimum Hamming distance. This distance is the Hamming distance of the complete code.

Assume two codewords: 10001001 and 10110001. This part determine how many bits are related differently. In this case, there are three different bits. To determine just do EXCLUSIVE OR operation on the second codeword, and count the number of 1 bits in operating results. Hamming distance is the number of bit positions in which two codewords differ. The thing to note is that when two separate codewords with Hamming distance d, it will take a single bit error $\mathrm{d}$ to convert from the one into the other. The properties of error detection and error recovery of a code depend on its Hamming distance. To detect d errors, it needs a code with $d+$ 1 because of the code as it was unlikely that $d$ single-bit errors can change a valid codeword into another valid codeword. 
When the recipient sees an invalid codeword, there has been an error in transmission. Likewise, to correct d errors, it needs a code within $2 d+1$ because it was declared legal codeword can be separated even with d changes, the original codeword will be closer than the other, then the error recovery can be determined uniquely.

As a simple example for error detection code, download a code in which a single parity bit is added to the data. The parity bit is chosen so that the number of 1 bits in the codeword becomes even (or odd). For example, if 10110101 is delivered in even-numbered parity by adding a bit at the edges, then the data was 101101011, while the even-numbered 10110001 parity becomes 101100010 . A code with a single parity bit has a distance of 2 because any single-bit error produces a codeword with parity wrong.

The following steps are done to calculate the hamming code as cited from Tim Downey from Florida International University [2]. The key to the Hamming Code is the use of extra parity bits to allow the identification of a single error. Create the codeword as follows:

- Mark all bit positions that are powers of two as parity bits. (positions 1, 2, 4, 8, 16, 32, 64, etc.)

- All other bit positions are for the data to be encoded. (positions 3, 5, 6, 7, 9, 10, 11, 12, 13, 14, 15, 17, etc.)

- Each parity bit calculates the parity for some of the bits in the code word. The position of the parity bit determines the sequence of bits that it alternately checks and skips.

- $\quad$ Position 1: check 1 bit, skip 1 bit, check 1 bit, skip 1 bit, etc. $(1,3,5,7,9,11,13,15, \ldots)$

- Position 2: check 2 bits, skip 2 bits, check 2 bits, skip 2 bits, etc. $(2,3,6,7,10,11,14,15, \ldots)$

- Position 4: check 4 bits, skip 4 bits, check 4 bits, skip 4 bits, etc. $(4,5,6,7,12,13,14,15,20,21,22,23, \ldots)$

- Position 8: check 8 bits, skip 8 bits, check 8 bits, skip 8 bits, etc. $(8-15,24-31,40-47, \ldots)$

- Position 16: check 16 bits, skip 16 bits, check 16 bits, skip 16 bits, etc. $(16-31,48-63,80-95, \ldots)$

- Position 32: check 32 bits, skip 32 bits, check 32 bits, skip 32 bits, etc. $(32-63,96-127,160-191, \ldots)$

Set a parity bit to 1 if the total number of ones in the positions it checks is odd. Set a parity bit to 0 if the total number of ones in the positions it checks is even.

\section{EVALUATION}

\subsection{Transmit}

This section describes the calculation of the Hamming code. Assume the data to transmit is the character of $\mathrm{H}$. The bit value of the character is 01001000 . Since the Hamming code uses 7 bits in this research, the bit value of $\mathrm{H}$ is 1001000 . The following calculation will explain how the character is transmitted.

$\begin{array}{ll}\text { Character } & : \mathrm{H} \\ \text { Decimal } & : \\ \text { Binary } & : 1001000\end{array}$

\begin{tabular}{|l|l|l|l|l|l|l|l|}
\hline Bit & 1 & 0 & 0 & 1 & 0 & 0 & 0 \\
\hline Position & 1 & 2 & 3 & 4 & 5 & 6 & 7 \\
\hline
\end{tabular}

The bit length is 7 represented by $p$.

$$
\begin{aligned}
\mathrm{p} & =7 \\
\mathrm{cb} & =2^{n}-(n-1)>7 \\
& =2^{1}-(1-1) \\
& =0<7 \\
\mathrm{cb} & =2^{n}-(n-1)>7 \\
& =2^{2}-(2-1) \\
& =1<7 \\
\mathrm{cb} & =2^{n}-(n-1)>7 \\
& =2^{3}-(3-1) \\
& =4<7 \\
\mathrm{cb} & =2^{n}-(n-1)>7 \\
& =2^{4}-(4-1) \\
& =11>7
\end{aligned}
$$

After the calculation, the total check-bits is 4 check-bits.

\begin{tabular}{|l|c|c|c|c|c|c|c|c|c|c|c|}
\hline Parity & $\mathbf{x}$ & $\mathbf{x}$ & 1 & $\mathbf{x}$ & 0 & 0 & 1 & $\mathbf{x}$ & 0 & 0 & 0 \\
\hline Position & 1 & 2 & 3 & 4 & 5 & 6 & 7 & 8 & 9 & 10 & 11 \\
\hline
\end{tabular}

There will be four check-bits must be inserted to the bit data The total length will be 11 bits (bitdata + bitparity). The bits are still unknown. There is a technique to replace the $\mathrm{x}$ with 0 or 1 .

Table 4. Data extraction

\begin{tabular}{|c|c|c|c|}
\hline Pos & Binary & Parity & Data \\
\hline 11 & 1011 & & 0 \\
\hline 10 & 1010 & & 0 \\
\hline 9 & 1001 & & 0 \\
\hline 8 & 1000 & $\mathrm{x}$ & \\
\hline 7 & 0111 & & 1 \\
\hline 6 & 0110 & & 0 \\
\hline 5 & 0101 & & 0 \\
\hline 4 & 0100 & $\mathrm{x}$ & \\
\hline 3 & 0011 & & 1 \\
\hline 2 & 0010 & $\mathrm{x}$ & \\
\hline 1 & 0001 & $\mathrm{x}$ & \\
\hline
\end{tabular}

Table 4 shows the extraction from the previous parity data. This step is to determine the value of $\mathrm{x}$ whether 0 or 1 . The method is to calculate how many " 1 " in each index of binary data (Parity not included). 
Table 5. Parities value

\begin{tabular}{|c|c|c|}
\hline Parity & Value & Amount \\
\hline x1 & 0 & there are 2 of 1 \\
\hline x2 & 0 & there are 2 of 1 \\
\hline x3 & 1 & There are 1 of 1 \\
\hline x4 & 0 & There are 0 of 1 \\
\hline
\end{tabular}

Table 5 show the value of every parity. There is rule to determine the value.If the amount is even then the value is 0 and if the amount is odd then the value is 1 .

Table 6. Data and parity

\begin{tabular}{|c|c|c|c|c|c|c|c|c|c|c|c|}
\hline Position & \multicolumn{11}{|c|}{ Insertion } \\
\hline 1 & $\mathbf{x 1}$ & 1 & 0 & 0 & 1 & 0 & 0 & 0 & & & \\
\hline 2 & $\mathbf{x 1}$ & $\begin{array}{l}x \\
2\end{array}$ & 1 & 0 & 0 & 1 & 0 & 0 & 0 & & \\
\hline 4 & $\mathbf{x 1}$ & $\begin{array}{l}\mathbf{x} \\
2\end{array}$ & 1 & $\begin{array}{l}\mathbf{x} \\
\mathbf{3}\end{array}$ & 0 & 0 & 1 & 0 & 0 & 0 & \\
\hline 8 & $\mathbf{x 1}$ & $\begin{array}{l}x \\
2\end{array}$ & 1 & $\begin{array}{l}x \\
3 \\
\end{array}$ & 0 & 0 & 1 & $x 4$ & 0 & 0 & 0 \\
\hline Result & 0 & 0 & 1 & 1 & 0 & 0 & 1 & 0 & 0 & 0 & 0 \\
\hline
\end{tabular}

Table 6 shows the final result of the data and parity formation. There are 11 bits now after the parity addtion.

$$
\text { New Binary : } 00110010000
$$

\subsection{Receive}

This section tries to detect and fix the error from previous binary string. The following calculation describes the full explanation of the Hamming. Assume that the error is set to the fifth bit.

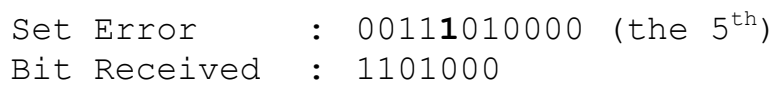

\begin{tabular}{|l|l|l|l|l|l|l|l|}
\hline Bit & 1 & 1 & 0 & 1 & 0 & 0 & 0 \\
\hline Position & 1 & 2 & 3 & 4 & 5 & 6 & 7 \\
\hline
\end{tabular}

Table 7. Data extraction

\begin{tabular}{|c|c|c|c|}
\hline Pos & Binary & Parity & Data \\
\hline 11 & 1011 & & 0 \\
\hline 10 & 1010 & & 0 \\
\hline 9 & 1001 & & 0 \\
\hline 8 & 1000 & $\mathrm{x}$ & \\
\hline 7 & 0111 & & 1 \\
\hline 6 & 0110 & & 0 \\
\hline 5 & 0101 & & 1 \\
\hline 4 & 0100 & $\mathrm{x}$ & \\
\hline 3 & 0011 & & 1 \\
\hline 2 & 0010 & $\mathrm{x}$ & \\
\hline 1 & 0001 & $\mathrm{x}$ & \\
\hline
\end{tabular}

Table 7 shows the extraction from the previous parity data.
Table 8. Parities value

\begin{tabular}{|c|c|c|}
\hline Parity & Value & Amount \\
\hline x1 & 1 & there are 3 of 1 \\
\hline x2 & 0 & there are 2 of 1 \\
\hline x3 & 0 & There are 1 of 1 \\
\hline x4 & 0 & There are 0 of 1 \\
\hline
\end{tabular}

Table 8 show the value of every parity. The value is different from the first before the error. Until now, it is proved there was a modification of the bit value. It means the data is not retrieved perfectly.

Table 9. Error position

\begin{tabular}{|c|c|c|}
\hline T. Parity & R. Parity & Error Pos \\
\hline 0 & 1 & 1 \\
\hline 0 & 0 & 0 \\
\hline 1 & 0 & 1 \\
\hline 0 & 0 & 0 \\
\hline
\end{tabular}

Error Position : 1010

Position Index : 5

The Hamming code detected the error in the fifth position as determined previously. It is proved that the method works to a significant condition.

\section{CONCLUSION}

By the test results and discussion programs that do, it can be concluded that the method of Hamming code can detect bit errors in the file received by the receiver file. The checking is done by comparing the value of the check bits with the original value of bits that exist in the file. Methods hamming code can make corrections damage bits as a bit, or it is called single bit error detection. It is the unfortunate method of Hamming code can not detect if there is more than one-bit error. Error detected just as much as the pieces only error even though possessed more than one.

\section{FUTURE SCOPE}

The next research is needed to expand the single error parity checking to the multi-error parity checking. The Hamming code is limited to one error only. If multi-error or burst error happens, Hamming can only detect the single error. By composing the block system, it lets the computer program perform the multi-error checking.

\section{REFERENCES}

[1] R. S. Geiger and A. Lampinen, "Old Against New, or a Coming of Age? Broadcasting in an Era of Electronic Media," Journal of Broadcasting \& Electronic Media, vol. 58, no. 3, pp. 333-341, 2014.

[2] T. Downey, "Calculating the Hamming Code," Florida International University, 252 2016. [Online]. Available: http://users.cis.fiu.edu/ downeyt/cop3402/hamming.html . [Accessed 110 2016].

[3] K. P. Debalina Roy Choudhury, "Design of Hamming Code Encoding and Decoding Circuit Using Transmission Gate Logic," International Research Journal of Engineering and Technology, vol. 2, no. 7, pp. 1165-1169, 2015. 
[4] E. S. Abuelyaman and A.-A. S. Al-Sehibani, "Optimization of the Hamming Code for Error Prone Media," International Journal of Computer Science and Network Security, vol. 8, no. 3, pp. 278-285, 2008.

[5] R. W. Hamming, "Error Detection and Error Correction Codes," Bell Systems Tech. Journal, vol. 29, no. 1, pp. 147-160, 1950.

[6] Z. Ramadhan and A. P. U. Siahaan, "Stop-and-Wait ARQ Technique for Repairing Frame and Acknowledgment Transmission," International Journal of Engineering Trends and Technology, vol. 38, no. 7, pp. 384-387, 2016.

[7] N. Shep and P. Bhagat, "Implementation of Hamming code using VLSI," International Journal of Engineering Trends and Technology, vol. 4, no. 2, pp. 186-190, 2013.

[8] D. R. Choudhury and K. Podder, "Design of Hamming Code Encoding and Decoding Circuit Using Transmission Gate Logic," International Research Journal of Engineering and Technology, vol. 2, no. 7, pp. 1165-1169, 2015.

[9] H. Saleh, "Design of Hamming Encoder and Decoder Circuits For $(64,7)$ Code and $(128,8)$ Code Using VHDL," Journal of Scientific and Engineering Research, vol. 2, no. 1, pp. 1-15, 2015.

[10] S. Cha and H. Yoon, "Efficient Implementation of Single Error Correction and Double Error Detection Code with
Check Bit Precomputation for Memories," Journal of Semiconductor Technology and Science, vol. 12, no. 4, pp. 418-425, 2012.

\section{AUTHOR PROFILE}

Wirda Fitriani was born in Medan, Indonesia, in 1979. She received the S.Kom. degree in computer science from Universitas Pembangunan Panca Budi, Medan, Indonesia, in 2005, She joined the Department of Engineering, Universitas Pembangunan Panca Budi, as a Lecturer in 2008, and in 2014, she entered the post-graduate university, AMIKOM, Yogyakarta. She is now studying for her master degree. She has become a writer in several conferences. She is still working as a System Support at PT. Buana Varia Komputama, Medan.

Andysah Putera Utama Siahaan was born in Medan, Indonesia, in 1980. He received the S.Kom. degree in computer science from Universitas Pembangunan Panca Budi, Medan, Indonesia, in 2010, and the M.Kom. in computer science as well from the University of Sumatera Utara, Medan, Indonesia, in 2012. In 2010, he joined the Department of Engineering, Universitas Pembangunan Panca Budi, as a Lecturer, and in 2012 became a researcher. He is applying for his $\mathrm{Ph} . \mathrm{D}$. degree in 2016. He has written several international journals. He is now active in writing papers and joining conferences. 